\title{
Geometry Systems for Lattice-Based Reconfigurable Space Structures
}

\author{
Megan Ochalek \\ Department of Mechanical Engineering \\ Massachusetts Institute of Technology \\ Cambridge, MA \\ mochalek@mit.edu
}

\author{
Benjamin Jenett \\ Center for Bits and Atoms \\ Massachusetts Institute of Technology \\ Cambridge, MA \\ bej@mit.edu
}

Olivia Formoso, Christine Gregg,

Greenfield Trinh, Kenneth Cheung

Coded Structures Laboratory

NASA Ames Research Center

Moffett Field, CA

kenny@nasa.gov

\begin{abstract}
We describe analytical methods for the design of the discrete elements of ultralight lattice structures. This modular, building block strategy allows for relatively simple element manufacturing, as well as relatively simple robotic assembly of low mass density structures on orbit, with potential for disassembly and reassembly into highly varying and large structures. This method also results in a structure that is easily navigable by relatively small mobile robots. The geometry of the cell can allow for high packing efficiency to minimize wasted payload volume while maximizing structural performance and constructability. We describe the effect of geometry choices on the final system mechanical properties and automated robotic constructability of a final system. Geometric properties considered include number of attachments per voxel, number of attachments per coefficient of volume, and effects of vertex, edge, and face connectivity of the unit cell. Mechanical properties considered include strength scaling, modulus scaling, and packing efficiency of the lattice. Automated constructibility metrics include volume allowance for an end-effector, strut clearance angle for an endeffector, and packing efficiency. These metrics were applied to six lattice unit cell geometries: cube, cuboctahedron, octahedron, octet, rhombic dodecahedron, and truncated octahedron. A case study is presented to determine the most suitable lattice system for a specific set of strength and modulus scaling requirements while optimizing for ease of robotic assembly.
\end{abstract}

\section{TABLe OF Contents}

1. INTRODUCTION........................... 1

2. BACKGROUND $\ldots \ldots \ldots \ldots \ldots \ldots \ldots \ldots \ldots \ldots \ldots \ldots$

3. Methodology $\ldots \ldots \ldots \ldots \ldots \ldots \ldots \ldots \ldots \ldots \ldots \ldots \ldots \ldots \ldots$

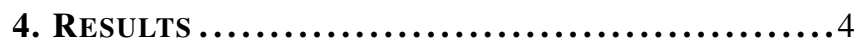

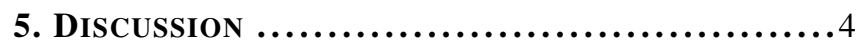

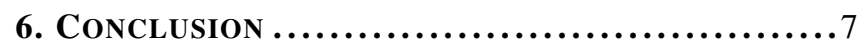

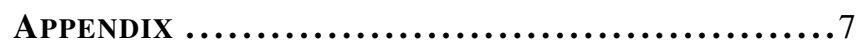

ACKNOWLEDGMENTS .......................... 7

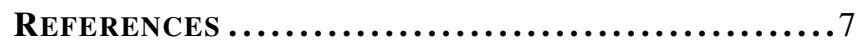

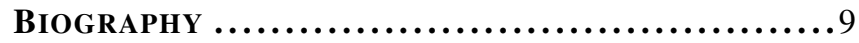

\section{INTRODUCTION}

This paper describes analytical methods for design of the discrete elements of assembled ultralight lattice structures. These modular building block based structures are intended

U.S. Government work not protected by U.S. copyright to enable relatively simple robotic assembly of low massdensity structures with the capability to be disassembled and reassembled into varying configurations. These structures are envisioned to have broad utility, including as the primary structure for various applications including kilometer scale telescope arrays [1], large space habitats [2], spacecraft structures, or even as adaptable emergency ground structures.

The objective of this work is to investigate candidate cell geometries and to assess their suitability for local/relative reference robot assembly. This is accomplished by analyzing relevant metrics such as mechanical performance, manufacturing methods, and effects on robot locomotion, to provide baseline data to inform design choices for the cell geometry of an integrated robotically assembled lattice structural system.

A brief literature review of cellular solids and automated manufacturing technologies is presented, followed by the methodology and metrics. Results for the study are presented as well as a discussion of the findings and lessons learned.

\section{BACKGROUND}

\section{Cellular Solids}

The field of cellular solids encompasses natural cellular materials such as bones, wood, and cork which have stochastic cells, as well as engineered cellular solids such as foams, architected lattice structures, and honeycomb core sandwich panels. They are characterized by an open substructure, very light mass density, and high surface area, often displaying multi-functional characteristics. Unit cell geometry of the substructure, together with constituent material, governs the resulting mechanical performance of the bulk lattice. [3] $\mathrm{Pi}$ oneering work by Gibson and Ashby has established that the stiffness and strength of any cellular solid are, respectively:

$$
\begin{aligned}
& E \propto \rho^{\alpha} \\
& \sigma \propto \rho^{\beta}
\end{aligned}
$$

where $\rho$ is the local/relative reference density (lattice density normalized by constituent material density), and $\alpha$ and $\beta$ are geometry dependent constants greater than or equal to 1 . An ideally performing lattice has $\alpha$ and $\beta$ approximately equal to 1.

Complex geometries associated with cellular materials 
present many manufacturing challenges, but recent advances in additive manufacturing (i.e., 3D printing) have given researchers new tools to carefully design lattice geometry. These materials are generally classified as architected materials, and have demonstrated near-ideal performance [4-6]. There are various typical fabrication methods, including twophoton lithography direct laser writing of hollow tube alumina nano-lattices [7]. Zheng et al. has shown the fabrication of a record ultra-light and ultra-strong microlattice on the centimeter scale using projection microstereolithography [8]. Such local/relative reference performance can also be achieved through polyjet 3D printing with print resolution on the micrometer scale [9]. While these lattices provide outstanding mechanical performance, the manufacturing size limits of these processes restrict these monolithic structures to the nano- to centimeter scale.

Recent work in discretely assembled lattice structures demonstrates a strategy to scalably manufacture architected lattice structures with comparable absolute mechanical performance to those made with additive manufacturing. Unlike 2D sheet-based assembled fabrication [10], discretely assembled lattices are a system of modular unit cells repeated in $3 \mathrm{D}$ to create larger structures. Using these unit cells, termed voxels, to build a larger working structure is part of the field described by Cheung and Gershenfeld as "digital materials" [4]. There are many advantages to this process, including an unlimited build envelope, reconfigurability, and tunability. These systems have been demonstrated in a variety of applications, including as the substructure for a morphing, twisting plane wing [11]. Jenett et al. used unit cells made of out of unidirectional carbon fiber tubes and injection molded joints to create bridges and structures that could support the weight of a human [12]. Fully injection molded unit cells at ultralight densities have been demonstrated by Gregg et al. [6].

\section{Discrete, Robotic Assembly}

The unlimited build envelope and opportunity for error correction and repair [6] suggest discrete lattice assembly systems as a viable strategy for creating meter scale (or larger) structures that utilize the outstanding mechanical performance of architected materials. However, to truly enable scalability in space applications, automated robotic assembly is highly desirable due to the high number of connections and limitations of current EVA processes [13]. Due to the discrete, repeating nature, lattice systems in general are well suited for large scale robotic assembly [14]. Though not categorized as cellular materials, automated manufacturing systems for truss assembly have been previously explored for space applications. The Automated Structural Research Lab at NASA Langley describes an autonomous truss assembler that constructs an eight meter diameter planar structure [15]. In the cellular and digital materials literature, gantry style robotic assemblers have been used to create lattice structures from node connected octahedra unit cells [16].

Limitations associated with conventional robotic assembly systems, such as a gantry or robot arm style assemblers, include build envelope limitations of the assembler and reliance on an global/absolute reference system of fixed envelope. Error identification and correction capability necessarily degrades beyond this envelope. With a goal of overcoming scaling problems associated with fixed global metrology to enable much larger scale lattice structures (such as kilometer scale), digital material assembly systems propose the utilization of local/relative reference and metrology based robots assembly robots that travel along periodic structures. Such systems should allow for extensive parallelization. Early examples of digital structure based local/relative reference based locomotion include the Modular Assembly System work conducted by Terada and Murata, with modular blocks that have integrated attachment mechanisms [17], and BILL$\mathrm{E}$, a bi-pedal inchworm robot that walks along the outside of a lattice structure [18].

Since local/relative reference robots are intended to locomote on the structure as well as to assemble it, the lattice geometry and robotic design are very closely integrated. Depending on the desired application and required lattice performance, we can choose an appropriate lattice geometry and design an associated robotic system. However, are there certain geometries that are more suitable for robotic assembly than others? In this study, we analyze prospective lattice geometries and attempt to evaluate the relationship between mechanical performance and ease of robotic assembly. In doing so, we seek to provide a framework for evaluating lattice and robotic systems to co-optimize lattice and robotic performance in the context of application constraints to yield a high performance, reliably automated, discrete lattice system.

\section{Methodology}

To determine the best suited geometry for a digital material lattice system, we defined metrics to compare potential candidates. These metrics were intended as tools for objective decision making for current and future digital lattice material designers. For this study, a comprehensive set of metrics was designed to assess the viability of the geometry as a high performance, robotically assemble-able voxel unit. This study allowed us to explore the advantages, disadvantages, and risks of each design. The end product of this analysis is a case study to determine geometries that appear to be well suited for large-scale local/relative reference robotic assembly.

\section{Geometry Candidates}

Lattice framework options were generated though the discrete affine translation of space-filling polyhedra. Also referred to as convex uniform honeycombs, these uniform tessellations fill three-dimensional space with non-overlapping convex uniform polyhedral cells. Each honeycomb consists of one or more types of polyhedra. These patterns were then divided into two groups: regular and heterogeneous. In this study, a lattice framework was referred to as "regular" if the tessellation is composed entirely of equal length struts and can be decomposed into a single repeating unit cell; that is, only one polyhedral unit cell geometry was needed to create the lattice. A "heterogeneous" lattice required more than one polyhedra unit cell type to tessellate the space.

Engineered periodic cellular structures are also represented in the literature from non-convex repeating units $[3,19]$, as well as with cell walls as closed cell structures [20]. Neither are considered for this study, for the purpose of limiting the complexity of the lattice and associated robotic assembly. Although recent results suggest that closed-cell lattices are more mechanically efficient, they were not considered here to enable internal cell access for robotic assembly, inspection, and locomotion operations.

We have compiled a list of convex polyhedra that tessellate regularly in 3D space, shown in Figure 5 in the Appendix. The branch of regular lattice frameworks is complete as far as is known, while the branch of heterogeneous frameworks lists only a small number of the possible tessellations that are 
known and described in the literature.

\section{Fixed Assumptions}

To bound the design problem, several assumptions were made regarding discrete unit cell joining methodology. Prior to the design study, a range of possible methods for the joining of the lattice structures were explored, including thermoplastic and thermoset polymers, mechanical joining, welding, adhesives, and molding. Although other methods may be used in creating high performance lattice structures, we focused in this study on mechanical joining due to its flexibility and known reliability through multiple attachment and detachment cycles [21]. Mechanical joints can range from threaded as bolts and nuts, to bayonet type, to complicated latching linkage systems and more. For the purposes of this paper, we assumed a captive generic threaded bolt and nut joint for vertex attached structures. Face and edge attachments were also explored during the course of this study, but we were unable to identify well characterized high strength linear or planar reversible mechanical attachment mechanisms in the literature (that were not otherwise well characterized as a finite set of discrete point connections). Incidentally, to remove the need for mechanical feeding systems in the robotic assembler, captive fastening mechanisms were treated as a requirement. We find that most mechanisms involving discrete mating parts are not difficult to implement as captive parts.

\section{Design Parameters}

To evaluate a given cell geometry against system requirements, design parameters were established. For any given application, thresholds and optimum values can be defined for each parameter to define system performance.

We define an adjacency as the location of neighboring voxels with respect to each other, occurring at either the face, edge, or vertex. An attachment is defined as the fastener location, which can in principle also be face, edge, or vertex. The nomenclature used here, to describe this complete set is in the format of Geometry-Adjacency-Attachment (e.g., CubeFace-Face), and is seen in Figure 1. Some of the given unit cell geometries can be employed in numerous ways to construct lattices, depending on their adjacency. Varying adjacency type for a given unit cell can alter the resulting lattice; varying attachment location changed the applicable types of fastening, and thus the robotic fastening method as well. Hence, we considered how various adjacency and attachment configurations influenced the dependent properties of the assembled lattice. For an attachment to be a true face attachment, it must spread the joining loads over an entire face. Since we do not consider closed cell geometry, it follows that we do not consider face attachment mechanisms (open cell face adjacency results in edge attachment as highest dimensional attachment type).

Number of attachments per voxel-The number of attachments per voxel is considered as a measure of the complexity of the unit cell.

Number of attachments per adjacency-The number of attachments per adjacency is also considered as a measure of ccell complexity, and is a function of adjacency and attachment types.

Coefficient of Volume-Coefficient of Volume is defined as the ratio of the volume contained within the convex hull of the voxel geometry to the space filling repeating volume that

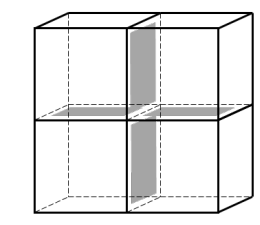

Cube - Face - Face
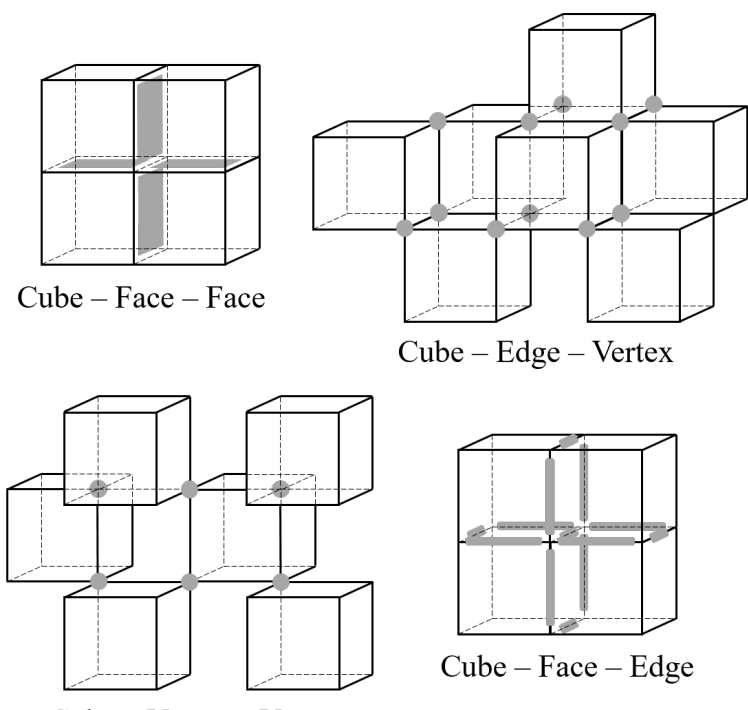

Cube - Vertex - Vertex

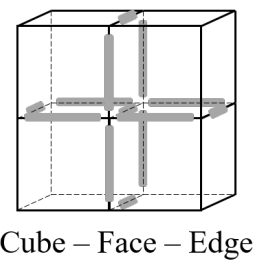

Figure 1. Examples of geometry configurations with attachment locations shown in gray. An adjacency refers to the location of a neighboring voxel with respect to the origin

voxel. An attachment is defined as the fastener location. Starting in the top left and moving clockwise, 1 and 3 are SC tiling, while 2 and 4 are BCC.

defines its assembly to the intended lattice geometry. For example, for a cuboct lattice, this can be either the volume of an octahedron to the volume of the cube enclosing that octahedron per simple cubic packing, or the volume of a cuboctahedron to the same.

Average number of attachments per coefficient of volumeThe average number of attachments per coefficient of volume as defined above.

\section{Geometric Effects (Dependent Properties)}

Stiffness and Strength Scaling-It is shown in the literature that stiffness and strength scaling are governed by the cell wall bending and can be determined using beam theory and dimensional analysis [3,22]. The resulting scaling laws with respect to the local/relative reference density are shown in equation 1 and 2 .

A few geometries are particularly well studied in the field of cellular solid materials. Where possible, empirical values for the stiffness and strength scaling were identified and cited herein. If experimental results were not available, computational estimations for the scaling laws were determined using the connectivity of the unit cell, as described by Deshpande et al. [23]. This provided a coarse estimation of the behavior of the lattice depending on whether the structure is transverse bend or axial stretch dominated, in terms of microstructural behavior under load.

Assembly Efficiency - We define assembly efficiency to include the cost and time of assembly, as well as the method (COTS vs custom), and the tolerances required.

Efficient assembly is seen as key to achieving efficient overall system. Assuming the consistent use of mechanical fasteners as stated earlier, the number of fasteners was considered to be a primary factor in determining assembly efficiency. 
Tiling-The tiling of the unit cell referred to the packing type of the unit cells once they were fastened to each other. Simple cubic packing (SC) occurred when consecutive layers of unit cells lay directly on top of the cells below them, i.e. simple cubic packing. Body centered cubic (BCC) tiling was defined as alternating layers of cells sitting offset to one another. This can be seen in Figure 1. Tiling and the spatial relationship of unit cells became important when characterizing the complexity of locomotion needed by the robot to move across the structure.

Packing efficiency - The packing efficiency relates the deployed volume (the volume of a completed lattice) to the payload volume of unassembled voxels.

Volume allowance for robotic end-effector-The robotic endeffector must be able to access all attachment points in order to join unit cells to each other. It is presumed that a larger volume allowance offers more range of motion to maneuver to each attachment. This was calculated by determining the volume ratio of the largest clearance volume for the endeffector to enter and exit the unit cell to the total volume of the cell. This end-effector clearance volume can be visualized in Figure 2.

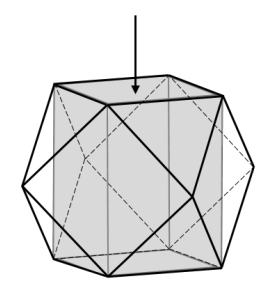

Cuboctahedron

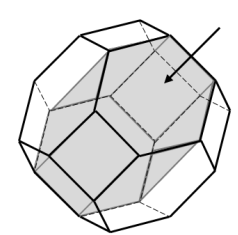

Truncated Octahedron

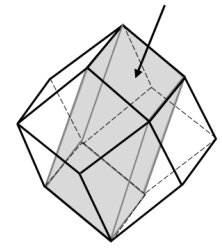

Rhombic

Dodecahedron
Figure 2. Images of end-effector clearance volume for some unit cells with arrows indicating which direction the end-effector enters the unit cell. The clearance volume is the largest volume for an and effector for enter and exit a cell for assembly. The ratio of the clearance volume and the total volume of the unit cell defines the volume allowance for the end-effector.

Strut Clearance Angle-The strut clearance angle, $\theta$, was another metric related to end-effector clearance. It was defined as the angle between the vector orthogonal to the node and the adjacent strut, as shown in Figure 3. A larger angle provided more clearance for the end-effector to operate near the node.

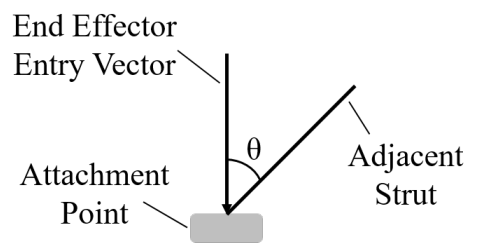

Figure 3. The strut clearance angle is defined as the angle between the end-effector entry vector, the vector orthogonal to the node, and the nearest adjacent strut.

\section{RESULTS}

The polyhedra studied in this analysis, shown in Figure 4, are:
- Cube

- Cuboctahedron

- Octahedron

- Octet

- Rhombic Dodecahedron

- Truncated Octahedron

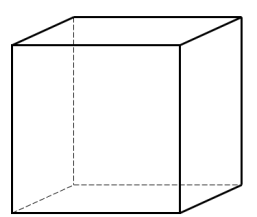

Cube

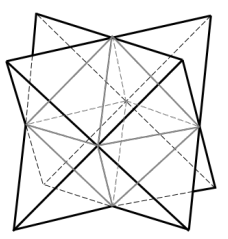

Octet

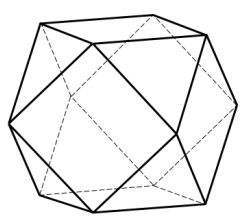

Cuboctahedron

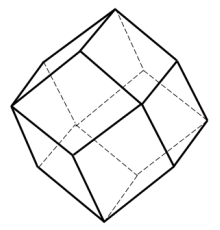

Rhombic

Dodecahedron

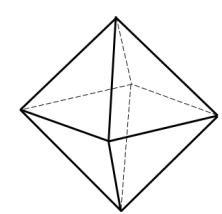

Octahedron

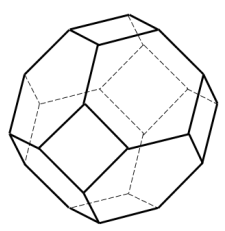

Truncated

Octahedron
Figure 4. Images of chosen polyhedral unit cells for analysis.

The candidates were then divided by their geometry configurations, i.e., the types of adjacencies and attachments that the polyhedra can have in order to create their resulting lattice. The independent properties described in the Methodology section above are listed in Table 1 . These include the number of attachments per voxel, number of attachments per adjacency, average number of attachments per coefficient of volume (COV), and symmetry type. The truncated octahedron and rhombic dodecahedron have significantly higher numbers of attachments per voxel. The rhombic dodecahedron and the cube had the highest average number of attachments per COV. All of the geometries had four fold symmetry.

The dependent metrics for the selected candidates are shown in Table 2. These include the stiffness and strength scaling, tiling, packing efficiency, volume allowance for an endeffector, and strut clearance angle for an end-effector. The cuboctahedron, octahedron, octet, and truncated octahedron scaling factors are found in the literature. About half of the geometry candidates are found to have simple cubic tiling. While the cube shows the highest volume allowance for an end-effector, it also provides the least amount of clearance, as will be explained in the following Discussion section. The octet unit cell shows zero volume allowance for an endeffector due to its complex shape and the presence of interior struts.

\section{DISCUSSION}

\section{Metrics Discussion}

The particular weighting of various metrics in evaluating geometries are application specific, but the compilation in this study provides insight into how candidate geometries affect robotic assembly and mechanical performance. Though most structural applications optimize for high specific strength and stiffness, the sufficiency of any geometry scaling is application dependent. For example, energy absorption applications may desire quadratic scaling materials with bending domi- 
Table 1. Independent Properties

\begin{tabular}{|c|c|c|c|c|c|}
\hline Geometry & $\nabla^{2}$ & $\nabla$ & $\hat{2}^{\circ}$ & $\gtrless^{\circ}$ & $\nabla^{\infty}$ \\
\hline Cube & Face & Edge & 24 & 4 & 12.0 \\
\hline Cube & Face & Vertex & 24 & 4 & 12.0 \\
\hline Cube & Edge & Edge & 12 & 1 & 6.00 \\
\hline Cube & Edge & Vertex & 24 & 2 & 12.0 \\
\hline Cube & Vertex & Vertex & 8 & 1 & 4.00 \\
\hline Cuboctahedron & Face & Edge & 24 & 4 & 5.09 \\
\hline Cuboctahedron & Face & Vertex & 24 & 4 & 5.09 \\
\hline Cuboctahedron & Vertex & Vertex & 12 & 1 & 2.55 \\
\hline Octahedron & Vertex & Vertex & 6 & 1 & 6.37 \\
\hline Octet & Edge & Edge & 24 & 1 & 4.26 \\
\hline Octet & Edge & Vertex & 30 & 1.25 & 5.32 \\
\hline Rhombic Dodecahedron & Face & Vertex & 12 & 4 & 7.79 \\
\hline Truncated Octahedron & Face & Edge & 72 & 5.14 & 2.83 \\
\hline Truncated Octahedron & Face & Vertex & 72 & 5.14 & 2.83 \\
\hline
\end{tabular}

nated microstructural behavior.

To simplify the assembly process and increase reliability, a reduction in the number of robotic processes is advantageous. A lower number of attachments per voxel indicates a lower number of fasteners (a decrease in requirement for robotic fastening) and likely faster assembly times. This measurement was also normalized by the number of adjacencies per voxel, as well as the coefficient of volume to better capture how the attachment quantities propagate across a completed lattice. The data presented in Table 1 shows that there are several geometries that exhibit a much lower number of attachments per voxel compared to others. Vertex-vertex geometry configuration are advantageous in this aspect as they result in a low number of attachments per voxel. On the other hand, a geometry that has a higher number of attachments, greater robotic assembly requirements, and slower assembly times may but not necessarily result in higher specific strength and stiffness. It should be noted that there may be mechanical solutions to actuate multiple attachments at the same time.

Unit cell geometry, when paired with adjacency type, affects the robotic placement motions needed to assemble a lattice. When placing a voxel in the proper position (such that it may be fastened and become part of the structure), interference with pre-placed voxels is a concern. This results in a number of placement motions needed for non-interference. It is expected that more placement motions will require more degrees of freedom and more mechanisms. This is expected to increase weight and control parameters, increasing system complexity and failure modes. Certain geometry configura- tions, such as Cuboctahedron-Vertex-Vertex, present particularly difficult placement and interference when alignment features are incorporated. Regular polyhedra that exhibit reflectional and 4-fold symmetry have many simplifying benefits to a digital material system as well. The orientation of the cell during system assembly is not limited to a single option, this property helps simplify robotic assembly and fastening by removing additional placement motions.

The existence of alignment features ideally allows for an imprecise assembler (here, local/relative reference robots) to assemble precisely, affecting system reliability. Adjacencies can be used as purely geometric alignment features to aid robotic assembly. As alignment feature size increases local/relative reference to the unit cell size, accuracy also increases; thus edge adjacencies have a greater alignment potential than vertex adjacencies.

We consider access to the interior of a voxel structure by the robot as essential for activation of the attachment without considerably mass expensive mechanisms. The volume allowance, shown in Table 2, is intended to provide insight into interference and trajectories that a robot may encounter. Higher volume allowances reduce risk for collisions, catching, and other unexpected issues that may occur during the assembly process. The cube geometry provides the maximum volume allowance, while the octet geometry, due to the internal strut interference, has a volume allowance of zero. Another metric that affects the end-effector clearance is the strut clearance angle, shown in Table 2. A larger angle reduces interference and risk. In the case of the Cube- 


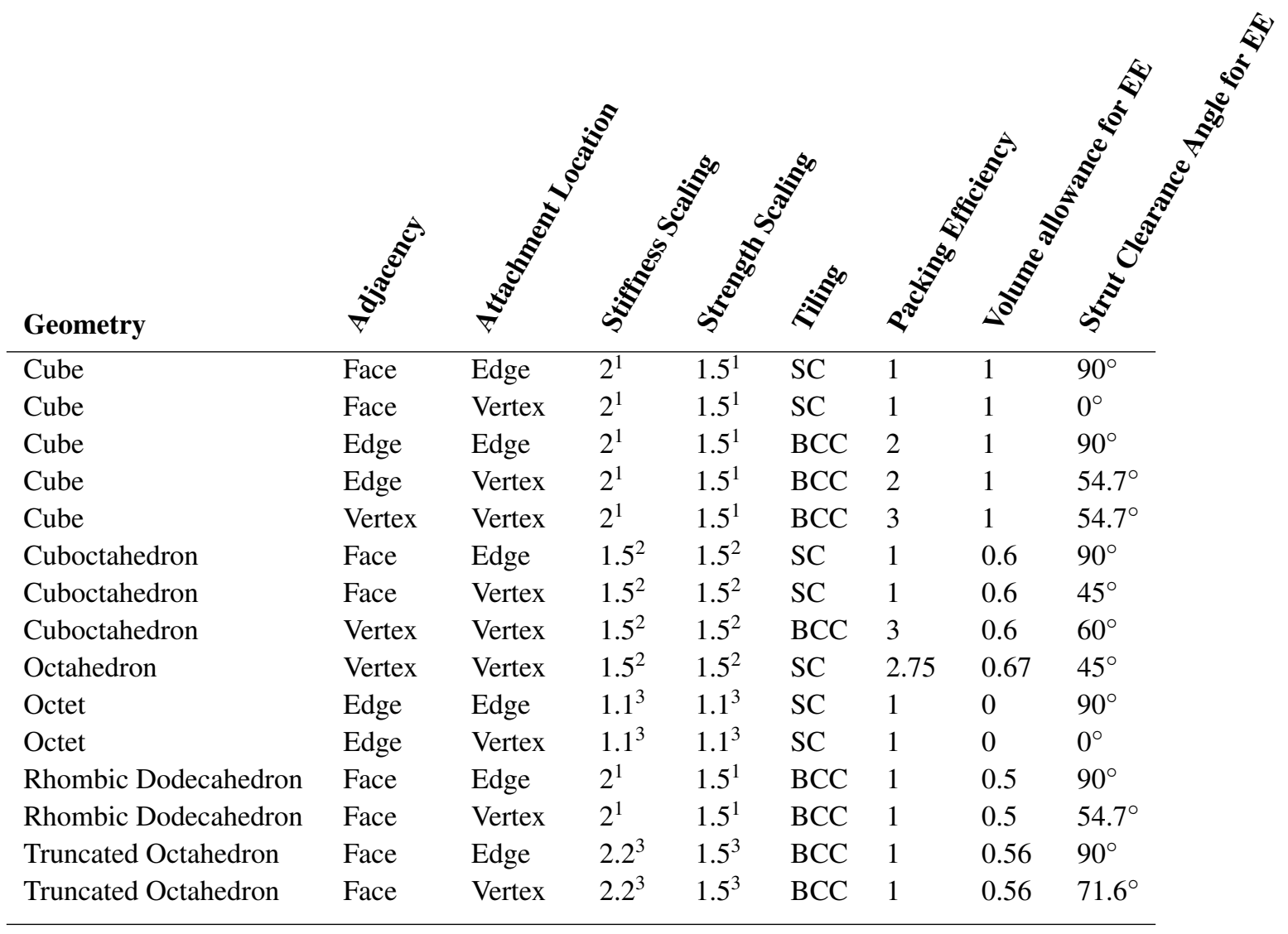

${ }^{1}$ Scaling factor was estimated due to the connectivity of the unit cell [23].

${ }^{2}$ Scaling factor was experimentally determined by Gregg et al. [6].

${ }^{3}$ Scaling factor was experimentally determined by Zheng et al. [5].

Face-Vertex and Octet-Edge-Vertex geometry, the vertex joint is located in an orientation where the strut is in line with the entry vector of the end-effector, requiring an orthogonal component to either the strut or the fastener direction.

The aforementioned metrics apply to both global/absolute reference and local/relative reference robotic assembly. In the case of a local/relative reference robot assembly system, there are additional criteria to consider when evaluating the cell geometry. Since the local/relative reference robot assembly system consists of the locomotion system as well as the assembly system, certain aspects of the cell geometry affect how the robot traverses across the lattice structure. For instance, the tiling of unit cells affects the types and number of motion primitives necessary to traverse the structure. SC tiling of unit cells, compared to BCC tiling, simplifies robotic locomotion down to the three XYZ coordinate axes. In locomotion through a $\mathrm{BCC}$ tiling lattice, the robot must traverse extra distance in order to reach the neighboring voxel.

Additionally, as the local/relative reference assembly robot transverses the lattice that it is building, it imparts local load concentrations on the structure. While robot locomotion loading is reduced in a microgravity environment, it may result in a damaged or deformed cell in a $1 \mathrm{G}$ environment. The impact of these loads on the structure and the assembly process depend on how the voxel is connected to the rest of the lattice. Having a higher number of attachments per adjacency, shown in Table 1 , increases this connectivity between voxels, thereby lessening the severity of the robotic loads effects by providing pathways to react to moment loads. However, as previously discussed, increasing the connectivity of a structure will increase robotic assembly complexity, so this should be balanced with locomotion and path planning requirements.

Packing efficiency, the essential factor in minimizing the transport volume of the pre-assembled system, is contingent on the geometry of the unit cell and the adjacency of the ensuing lattice. This property is shown in Table 2 . In order for a packing efficiency to be greater than one, there must exist a packing orientation more efficient than the assembled lattice itself. This existence depends on the density of the assembled lattice (if holes exist in comparison to that unit cells full lattice) as well as the negative space of the full lattice. Such holes exist when a unit cell can have multiple adjacency types; moving from face to edge to vertex adjacency increases the quantity of the holes. Additionally, if the repeating negative space of a full lattice is greater than the volume of the unit cell, it is implied that packing efficiency is greater than one. Packing efficiency can be increased even further by breaking down a unit cell into smaller packable components, but characterization of this option was outside the scope of the present study. 


\section{Prototypical Selection Process}

As a prototypical example, we select here for geometries that optimize the reliability and ease of local/relative reference robot assembly while maintaining the highest possible mechanical properties. For this down selection, time of robotic assembly is not a concern, but reliability and ease of robotic assembly are highest priority. Metrics that simplify robotic locomotion such as low total number of attachments and endeffector clearance are also of high priority. Stiffness and strength scaling and packing efficiency are important metrics, but are not as high of a concern.

In our case, the selection process for determining the best suited geometry for robotic assembly follows a process of elimination method. From the tree of possible candidates, Fig. 5, regular non-heterogeneous geometries that meet the minimum material performance specifications, or are very close, are selected for further study. In the next pass of the elimination process, candidate geometries are evaluated and selected via the above metrics. Geometry configurations that are non-simple cubic tiling are eliminated due to the added complexity in locomotion and voxel placement that they added. Geometry configurations that have zero volume allowance for the end-effector or have strut interference at the joints are also eliminated. Configurations with a high number of attachments are also eliminated, and the resulting geometries below are chosen for the next phase.

The resulting geometries include:

1. Cube-Face-Vertex

2. Cube-Face-Edge

3. Cube-Face-Face

4. Cuboctahedron-Face-Vertex

5. Cuboctahedron-Face-Edge

6. Cuboctahedron-Face-Face

7. Octahedron-Vertex-Vertex

In the final phase of the down selection, the questions that need to be answered are related to the design of face and edge attachments and the effects of local/relative reference robot locomotion and assembly on the structure. Face and edge attachment mass scale much higher than vertex attachment mass when multiple designs are evaluated; this leads to the elimination of those geometry configurations. In evaluating the remaining geometry configurations for compatibility with local/relative reference robot locomotion, configurations that have at least three attachments per adjacency are advantageous because they allow locomotion on the newly attached structure directly after attachment. This results in the Cubocahedron-Face-Vertex geometry being the remaining candidate, and therefore the best suited geometry for local/relative reference robot assembly.

Cuboctahedron-Face-Vertex-The Cuboctahedron-Face-Vertex configuration does not have the lowest total attachment configuration or highest mechanical performing geometry, but the benefits of having a moment resisting structure from just one additional unit cell greatly reduces the locomotion and path planning requirements for the system as a whole. Volume allowance and strut clearance angle is moderate compared to the other geometries.

\section{Conclusion}

Discrete lattice structures allow robotic assembly of large scale structures with high mechanical performance. We propose using autonomous assembly processes with modular unit cell building blocks to be able to build these structures on a larger scale. To develop a high performing structural system that is efficient for robotic assembly, multiple types of geometry were investigated for use as the structural building block for these lattice systems.

Using metrics catered to robotic assembly while still maintaining desired structural properties, various polyhedra were studied. Taking into account the stiffness and strength scaling values of the lattices led to a deeper study of how a cubic, cuboctahedral, and octahedral unit cell could be autonomously assembled. Metrics including the number of attachments per voxel, ability to form a moment-resisting structure, and volume allowance for a joining end-effector were evaluated. It was decided that the most suitable geometry configuration for a near term local/relative reference robot assembly project is Cuboctahedron-Face-Vertex. This geometry configuration demonstrated high mechanical performance, while also providing reasonable end-effector clearance, a moderate number of attachments, and helping simplify robotic locomotion requirements.

Future work for these systems includes analyzing optimal attachment schemes for the unit cells. This study was catered towards local/relative reference robotic assembly, and may be extensible to metrics and geometries for other digital material assembly systems, such as extrusion or gantry style assemblers. It is hoped that the comparisons made in this study will aid in the development of automated manufacturing for space applications.

For this study, we analyzed geometries that resulted in regular lattices. We further restricted the candidate geometries to those well studied in the field of cellular materials literature. Further constraints were accepted with the intent of reducing system complexity for robotic assembly and to assure that mechanical behavior metrics could be well characterized, but it is expected that success of near-term efforts will lead to future studies that relax these restrictions as robotic systems develop the capability to handle more complexity and as cellular mechanics literature progresses.

\section{APPENDIX}

\section{ACKNOWLEDGMENTS}

The authors thank the NASA Game Changing Development (GCD) Program for funding this work. We also thank Joseph Kim, Allan Costa, Irina Kostitsyna, and Afsheen Sajjadi for critical discussions.

\section{REFERENCES}

[1] C. Gregg, B. Jenett, D. Cellucci, and K. Cheung, "Design of multifunctional hierarchical space structures," in Aerospace Conference, 2017 IEEE. IEEE, 2017.

[2] D. Cellucci, B. Jennett, and K. Cheung, "Digital cellular solid pressure vessel: A novel approach for human habitation in space," in Aerospace Conference, 2017 IEEE. IEEE, 2017.

[3] L. J. Gibson and M. F. Ashby, Cellular solids: structure and properties. Cambridge university press, 1999.

[4] K. C. Cheung and N. Gershenfeld, "Reversibly assembled cellular composite materials," science, p. 1240889 , 


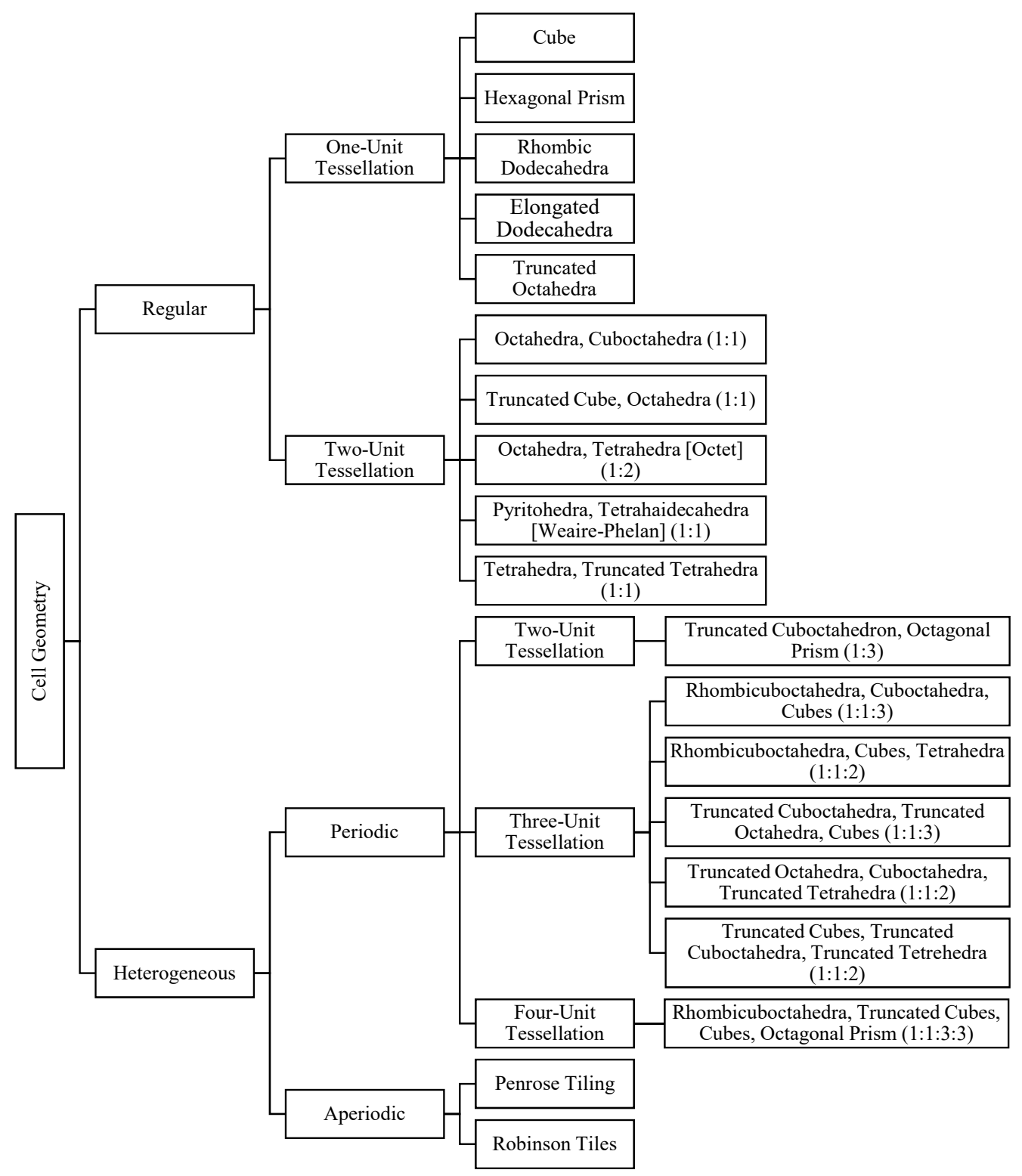

Figure 5. 3D tesselating polyhedra.

2013.

[5] X. Zheng, H. Lee, T. H. Weisgraber, M. Shusteff, J. DeOtte, E. B. Duoss, J. D. Kuntz, M. M. Biener, Q. Ge, J. A. Jackson et al., "Ultralight, ultrastiff mechanical metamaterials," Science, vol. 344, no. 6190, pp. 1373-1377, 2014.

[6] C. E. Gregg, J. H. Kim, and K. C. Cheung, "Ultra-light and scalable composite lattice materials," Advanced
Engineering Materials, 2018.

[7] L. R. Meza, S. Das, and J. R. Greer, "Strong, lightweight, and recoverable three-dimensional ceramic nanolattices," Science, vol. 345 , no. 6202, pp. 13221326, 2014.

[8] X. Zheng, W. Smith, J. Jackson, B. Moran, H. Cui, D. Chen, J. Ye, N. Fang, N. Rodriguez, T. Weisgraber et al., "Multiscale metallic metamaterials," Nature ma- 
terials, vol. 15, no. 10, p. 1100, 2016.

[9] M. Kaur, S. M. Han, and W. S. Kim, "Threedimensionally printed cellular architecture materials: perspectives on fabrication, material advances, and applications," MRS Communications, vol. 7, no. 1, pp. 819, 2017.

[10] L. Dong and H. Wadley, "Mechanical properties of carbon fiber composite octet-truss lattice structures," Composites Science and Technology, vol. 119, pp. 2633, 2015.

[11] B. Jenett, S. Calisch, D. Cellucci, N. Cramer, N. Gershenfeld, S. Swei, and K. C. Cheung, "Digital morphing wing: active wing shaping concept using composite lattice-based cellular structures," Soft robotics, vol. 4, no. 1, pp. 33-48, 2017.

[12] B. Jenett, D. Cellucci, C. Gregg, and K. Cheung, "Meso-scale digital materials: modular, reconfigurable, lattice-based structures," in ASME 2016 11th International Manufacturing Science and Engineering Conference. American Society of Mechanical Engineers, 2016, pp. V002T01A018-V002T01A018.

[13] J. J. Watson, T. J. Collins, and H. G. Bush, "A history of astronaut construction of large space structures at nasa langley research center," in Aerospace Conference Proceedings, 2002. IEEE, vol. 7. IEEE, 2002, pp. 7-7.

[14] G. Trinh, G. Copplestone, M. O'Connor, S. Hu, S. Nowak, K. Cheung, B. Jenett, and D. Cellucci, "Robotically assembled aerospace structures: Digital material assembly using a gantry-type assembler," in Aerospace Conference, 2017 IEEE. IEEE, 2017, pp. $1-7$.

[15] W. Doggett, "Robotic assembly of truss structures for space systems and future research plans," in Aerospace Conference Proceedings, 2002. IEEE, vol. 7. IEEE, 2002, pp. 3589-3598.

[16] O. Formoso, G. Trinh, S. Hu, and K. Cheung, "Development and robustness characterization of a digital material assembly system," Procedia Manufacturing, vol. 26, pp. 1003-1013, 2018.

[17] Y. Terada and S. Murata, "Automatic assembly system for a large-scale modular structure-hardware design of module and assembler robot," in Intelligent Robots and Systems, 2004.(IROS 2004). Proceedings. 2004 IEEE/RSJ International Conference on, vol. 3. IEEE, 2004, pp. 2349-2355.

[18] B. Jenett and K. Cheung, "Bill-e: Robotic platform for locomotion and manipulation of lightweight space structures," in 25th AIAA/AHS Adaptive Structures Conference, 2017, p. 1876.

[19] D. Cellucci and K. C. Cheung, "Evaluation of cellular solids derived from triply periodic minimal surfaces," in ASME 2016 11th International Manufacturing Science and Engineering Conference. American Society of Mechanical Engineers, 2016, pp. V002T01A020V002T01A020.

[20] J. Berger, H. Wadley, and R. McMeeking, "Mechanical metamaterials at the theoretical limit of isotropic elastic stiffness," Nature, vol. 543, no. 7646, p. 533, 2017.

[21] R. W. Messler, Joining of materials and structures: from pragmatic process to enabling technology. Butterworth-Heinemann, 2004.

[22] N. A. Fleck, "An overview of the mechanical properties of foams and periodic lattice materials," Cell Met Polym, pp. 3-7, 2004.

[23] V. Deshpande, M. Ashby, and N. Fleck, "Foam topology: bending versus stretching dominated architectures," Acta materialia, vol. 49, no. 6, pp. 1035-1040, 2001.

\section{BIOGRAPHY}

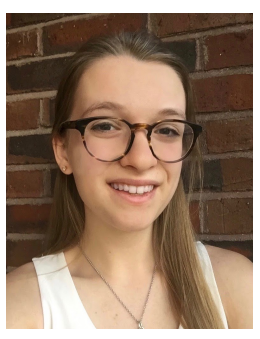

Megan Ochalek is currently pursuing her B.S. degree in Mechanical Engineering from the Massachusetts Institute of Technology. She interned at the NASA Ames Coded Structures Lab in 2018 working on automated assembly of digital material structures. At MIT, she has done research with the International Design Center, and is currently part of MIT EnergyHack.

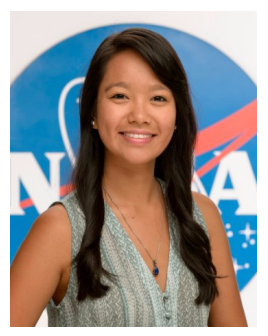

Olivia Formoso received her B.S. degree in Chemical Engineering from the University of Florida in 2016. Currently, she is a research engineer at the Coded Structures Lab at NASA Ames Research Center and is pursuing her M.S. in Mechanical Engineering at San Jose State University. Her research is focused on digital material structures and robotics.

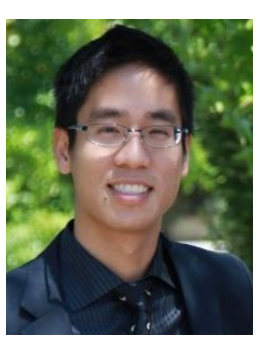

Greenfield Trinh is a research engineer in the Coded Structures Lab at NASA Ames Research Center. His current research activities include automated assembly of digital material structures and robotics. He received his B.S. in Physics from UC Riverside and M.S. in Aerospace Engineering from San Jose State University.

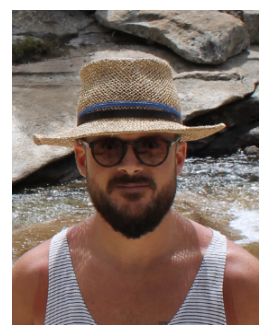

Benjamin Jenett is a graduate student researcher at the Massachusetts Institute of Technology's Center for Bits and Atoms, where he is pursuing his Ph.D. on automated assembly for large aerospace structures. He was formerly a NASA Space Technology Research Fellow and is currently in the NASA Pathways program. 


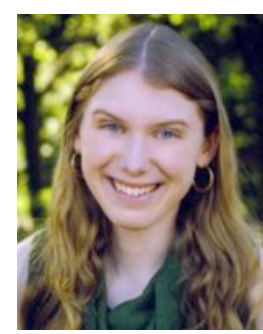

Christine Gregg received her Ph.D. from the Department of Mechanical Engineering at UC Berkeley, where she was a NASA Space Technology Research Fellow. Her thesis focused on digital lattice structures and lattice fracture mechanics. She works in the ARC Coded Structures Laboratory (CSL).

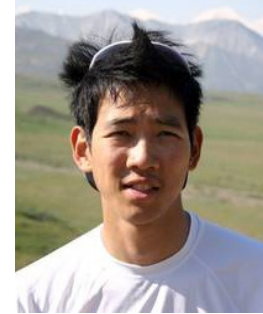

Kenneth Cheung received his Ph.D. from the Center for Bits and Atoms at the Massachusetts Institute of Technology. He helps to run the ARC Coded Structures Laboratory (CSL), which conducts research on the application of building block based materials and algorithms to aeronautical and space systems. As a member of the NASA ARC Intelligent Systems Division and affiliate of the office of the Center Chief Technologist, he serves as a technical lead on advanced materials and manufacturing. 